\title{
Custos hospitalares associados aos eventos adversos medicamentosos: Revisão
} sistemática

\author{
Hospital costs associated with adverse drug events: Systematic review \\ Costos hospitalarios asociados con eventos adversos de medicamentos: revisión sistemática
}

Recebido: 18/03/2021 | Revisado: 26/03/2021 | Aceito: 29/03/2021 | Publicado: 08/04/2021

Sara Cristina da Silva

ORCID: https://orcid.org/ 0000-0003-0005-3762 Universidade de Guarulhos, Brasil

E-mail: meggiesaraesabely@gmail.com

Rodrigo Corvino Rodrigues

ORCID: https://orcid.org/ 0000-0003-0421-9053 Universidade Estadual Paulista, Brasil

E-mail: eng.rodrigo.corvino@outlook.com

Meline Rossetto Kron Rodrigues

ORCID: https://orcid.org/0000-0003-2174-268X Universidade de Guarulhos, Brasil E-mail:me_kron@hotmail.com

\begin{abstract}
Resumo
Contexto: Os eventos adversos medicamentosos (EAMs) estão entre as causas mais rotineiras durante o processo de cuidado no ambiente hospitalar.É caracterizado como qualquer ocorrência clínica desfavorável que possa acometer o paciente durante o período de tratamento não sendo atribuída à evolução natural da doença de base. Objetivo: Analisar os custos da assistência prestada aos pacientes acometidos por EAM. Metodologia: Revisão sistemática com escrita pautada no check list PRISMA e Meta-analysis of Observational Studies in Epidemiology Statements (MOOSE) com consulta nas bases de dados da literatura PubMed,Embase,Cochrane e LILACS no mês de fevereiro de 2020. Os estudos foram selecionados a partir dos descritores controlados e seus sinônimos: "Efeitos Colaterais e Reações Adversas Relacionados a Medicamentos" e "Custos Hospitalares". O risco de viés foi analisado por meio da ferramenta Newcastle-Ottawa. Resultados: A análise incluiu oito estudos e evidenciou que há intenso aumento dos custos associados a ocorrência de EAM, bem como consequências negativas como aumento do tempo de internação, necessidade de mão de obra especializada e exposição a danos do paciente. Conclusão: Esta análise poderá ser um poderoso instrumento gerencial para as instituições de saúde como demonstrativo dos custos decorrentes dos EAMs, bem como fornece subsidio para pesquisas futuras.
\end{abstract}

Palavras-chave: Efeitos colaterais e reações adversas relacionados a medicamentos; Custos hospitalares; Custos e análise de custo; Economia da saúde.

\begin{abstract}
Context: Adverse drug events (AMEs) are among the most routine causes during the care process in the hospital environment. It is characterized as any unfavorable clinical occurrence that may affect the patient during the treatment period and is not attributed to the natural evolution of the underlying disease. Objective: To analyze the costs of care provided to patients affected by AMEs. Methodology: Systematic review with writing based on the PRISMA checklist and Meta-analysis of Observational Studies in Epidemiology Statements (MOOSE) with consultation in the PubMed, Embase, Cochrane and LILACS literature databases in February 2020. The studies were selected from the controlled descriptors and their synonyms: "Drug-Related Side Effects and Adverse Reactions" and "Hospital Costs" and for the English version, Mesh was used. The risk of bias was analyzed using the Newcastle-Ottawa tool. Results: The analysis included eight studies and showed that there is an intense increase in costs associated with the occurrence of AMI, as well as negative consequences such as increased length of hospital stay, the need for specialized labor and exposure to patient damage. Conclusions: This analysis can be a powerful managerial tool for health institutions as a demonstration of the costs arising from the AMS, as well as providing subsidies for future research.
\end{abstract}

Keywords: Drug-related side effects and adverse reactions; Hospital costs; Costs and cost analysis; Health economics.

\section{Resumen}

Marco contextual: Los eventos adversos por medicamentos (AMEs) se encuentran entre las causas más comunes durante el proceso de atención en el entorno hospitalario. Se caracteriza como cualquier acontecimiento clínico 
desfavorable que pueda afectar al paciente durante el período de tratamiento y no se atribuye a la evolución natural de la enfermedad de base. Objetivo: Analizar los costos de la atención brindada a los pacientes afectados por AME. Metodología: Revisión sistemática con redacción basada en el checklist PRISMA y Metaanálisis de Estudios Observacionales en Declaraciones Epidemiológicas (MOOSE) con consulta en las bases de datos de la literatura PubMed, Embase, Cochrane y LILACS en febrero de 2020. Los estudios fueron seleccionados de los descriptores controlados y sus sinónimos: "Efectos Colaterales y Reacciones Adversas Relacionados con Medicamentos" y "Costos de Hospital" y para la versión en inglés se utilizó Mesh. El riesgo de sesgo se analizó mediante la herramienta Newcastle-Ottawa.Resultados: El análisis incluyó ocho estudios y mostró que hay un aumento intenso en los costos asociados con la ocurrencia de IAM, así como consecuencias negativas como el aumento de la estancia hospitalaria, la necesidad de mano de obra especializada y la exposición al daño del paciente. Conclusión: Este análisis puede ser una poderosa herramienta de gestión para las instituciones de salud como demostración de los costos derivados de los AME, además de brindar apoyo para futuras investigaciones.

Palabras clave: Efectos colaterales y reacciones adversas relacionados con medicamentos; Costos de hospital; Costos y análisis de costo; Economía de la salud.

\section{Introduçãa}

A ocorrência dos eventos adversos (EA) é caracterizado como qualquer ocorrência clínica desfavorável que possa acometer o paciente durante o período de tratamento não sendo atribuída à evolução natural da doença de base e ocorrendo entre $4,0 \%$ a $16 \%$ das hospitalizações em países desenvolvidos, dentre os quais $60 \%$ podem ser preveníeis (Fernandes \& Queiros, 2011).

Todo procedimento assistencial envolve um risco de evoluir para um evento adverso, portanto fator potencial de risco, quase falha ou near-miss é qualquer variação de processo que pode acarretar chance significativa de desencadear um resultado negativo (Pena \& Meleiros, 2018). São condições, situações, procedimentos e condutas incertas que têm probabilidade de causar danos no paciente. Caso o dano ocorrer é denominado de evento adverso e resultará em efeito negativo sobre o paciente, ao colaborador, ao ambiente de trabalho e à organização (Moreira et al., 2015).

Os eventos adversos são classificados em eventos adversos infecciosos, correspondendo as Infecções Relacionadas à Assistência à Saúde (IRAS) que correspondem as infecções cuja aquisição está relacionada a um procedimento assistencial ou hospitalização, e Eventos Adversos Não Infecciosos, sendo os Eventos Adversos Não Infecciosos Relacionados à Assistência à Saúde, correspondendo a troca do local do procedimento cirúrgico, relacionada à medicação, quedas ou falha na identificação (Brasil, 2019).

Tendo em vista a perspectiva da assistência relacionada aos medicamentos, sabe-se que os medicamentos se tornaram uma importante ferramenta terapêutica, auxiliando na melhoria da qualidade e expectativa de vida da população, porém seu processo de administração de medicação é complexo e interdisciplinar, composto por três fases (prescrição, dispensação e administração) (Roque \& Melo, 2012).

No contexto intra-hospitalar, o processo da administração de medicação abarca a prescrição médica, dispensação realizada pelo farmacêutico e o preparo e administração da medicação realizada pela equipe de enfermagem. Quando há equívoco neste processo, cognominamos de evento adverso medicamentoso (EAM), ou seja, é caracterizado como um dano causado durante a administração da medicação. Deste modo, a EAM engloba toda e qualquer reação ruim ou não esperada que o paciente apresente após a administração de um medicamento (Abreu, Rodrigues \& Paixão, 2013).

Os EAMs estão entre as causas mais rotineiras durante o processo de cuidado no ambiente hospitalar e estima-se que 14,8\% a 59\% deles poderiam ser evitados. A literatura aponta que a maioria dos EAM não acarretam sequelas notáveis aos pacientes por ele acometido, porém alguns medicamentos podem induzir maior tempo de internação hospitalar, complicações, bem como a necessidade de novos exames e novos tratamentos, sequelas incapacitantes e até morte de pacientes (Fonteles et al., 2009; Abreu, Rodrigues \& Paixão, 2013)

Uma das causas mais manifestas do EAM são as reações adversas aos medicamentos (RAM) que ocorrem devido aos 
atributos intrínsecos das substâncias medicamentosas. A RAM é caracterizada como qualquer resposta prejudicial ou indesejável, não intencional, a um medicamento, que ocorre com o uso de doses usualmente utilizadas para profilaxia, diagnóstico ou terapia de doenças (Abreu, Rodrigues \& Paixão, 2013).

Na ocorrência da RAM, nota-se a existência de uma associação entre o uso do medicamento e a ocorrência do problema. Sendo assim, a RAM é definida como um tipo de evento adverso (Roque \& Melo, 2012).

Sabe-se que a segurança do paciente deve ser vista como um conjunto de estratégias/intervenções capazes de prevenir e reduzir o risco de dano ao paciente decorrente do cuidado em saúde, especialmente com o uso de medicações (Giordani et al., 2012).Especialistas em segurança do paciente em todo o mundo procuram identificar as medidas de segurança para evitar os erros relacionados com administração com medicamentos, que estão associados ao aumento da morbidade e mortalidade, além de refletir na economia de um país (Silva, Silva \& Pereira, 2016).

É de extrema importância a identificação dos custos gerado em consequência do evento adverso relacionado à medicação, pois permite ao gestor uma visibilidade do problema, visto que os recursos são escassos e finitos, necessitam otimizar de maneira eficiente nas instituições de saúde (Silva, Silva \& Pereira, 2016).

Um estudo realizado no Brasil identificou que entre as consequências dos danos ou lesões causadas ao paciente em decorrência da assistência, houve o aumento expressivos dos custos de internações hospitalares, permanência em hospitais, a necessidade de intervenção diagnóstica e terapêutica, os custos sociais, com aumento dos medicamentos, ausência no trabalho e morte prematura (Araújo \& Melo, 2017)

Sabe-se que a ocorrência de eventos adverso colabora para aumentar o tempo de internação, necessidade de exames complementares, mão de obra especializada e isso associa-se ao aumento dos custos hospitalares. Tal cenário interpõe a ocorrência de eventos adversos como um importante fator de impacto financeiro no Sistema Único de Saúde (SUS) por acarretar o aumento na morbidade, na mortalidade, no tempo de tratamento dos pacientes e nos custos assistenciais, além de repercutir em outros campos da vida social e econômica do país (Brasil, 2013).

A literatura interpõe por meio de um estudo descritivo e exploratório sobre a magnitude financeira associada à ocorrência de EAs em hospitais no Brasil, que os danos ao paciente decorrentes do cuidado à saúde têm expressivo impacto nos gastos hospitalares e que a importância financeira da ocorrência de eventos adversos, que, em parte, implicam em dispêndio de recursos desnecessários que poderiam ser utilizados para financiar outras necessidades de saúde da população. (Porto et al., 2010)

No Brasil, a atenção à saúde é um direito do cidadão e dever do estado, entretanto, os recursos são finitos e deve ser preocupação da academia propor a melhor e mais segura terapêutica ao menor custo corroborando para a necessidade de realização do presente estudo (Carvalho et al.,2020). Diante do exposto, a presente revisão sistemática objetiva identificar os custos hospitalares associados a ocorrência de evento adverso medicamentoso (EAM) disponíveis na literatura.

\section{Metodologia}

Trata-se de um estudo bibliográfico de revisão sistemática que seguiu as normativas do check list PRISMA (Moher et al., 2015) e Meta-analysis of Observational Studies in Epidemiology Statements (MOOSE) (Stroup et al., 2000).

\section{Critérios de Elegibilidade}

Foram consultadas as bases de dados eletrônicas sendo a National Center for Biotechnology Information (NCBI/PubMed), Embase, Cochrane Library e Literatura Latino-Americana e do Caribe em Ciências da Saúde (LILACS) no mês de fevereiro de 2020. A estratégia de pesquisa básica foi desenvolvida para PubMed e modificada conforme necessário para outras bases de dados (apêndice A). Foram utilizados os descritores em saúde disponíveis em Descritores em Ciências da 
Saúde (DECs) e no Medical Subject Heading (MeSH). Os descritores utilizados incluirão "Drug-Related Side Effects and Adverse Reactions", "Hospital Costs" e "Costs and Cost Analysis". Não houve restrição de idioma, mas apenas estudos em humanos foram selecionados. Referências dos artigos selecionados, incluindo artigos de revisão pertinentes, foram revisados para identificar todos os estudos relevantes. Foram incluídos estudos observacionais e de avaliação econômica que reportarem análise de custos associados a ocorrência de eventos adversos medicamentosos, seguindo a metodologia "PECO" descrita a diante; População: Pacientes em cuidado de saúde, Exposição: Ocorrência de Eventos adversos medicamentosos Comparador: Não ocorrência de eventos adversos medicamentosos. Outcomes: custos.

\section{Critérios de inclusão e exclusão}

A revisão sistemática avaliou os custos hospitalares em pacientes acometidos por eventos adversos medicamentosos. Estudos observacionais ou de avaliações econômicas que reportarem custos monetários decorrentes da ocorrência de eventos adversos medicamentosos foram incluídos. A exposição de interesse foi a ocorrência de eventos adverso medicamentoso vs. análise de custos. Os critérios de exclusão foram: estudos não reportarem custos associados a eventos adversos medicamentosos, estudos que reportem custos associados a eventos adversos não medicamentoso, estudos de revisão integrativa e de literatura, livros, capítulos e resenhas de livros, manuais, relatórios técnicos.

\section{Seleção dos estudos e extração de dados}

Para esta revisão, dois pesquisadores revisaram os títulos e resumos de elegibilidade de forma independente. Discordâncias em relação à seleção dos artigos foram resolvidas por consenso ou discussão com um terceiro investigador. O fluxograma de seleção dos estudos foi criado de acordo com as diretrizes PRISMA.

Dois pesquisadores avaliaram e extraíram independentemente os dados relevantes de cada artigo de texto completo utilizando formulário padronizado baseado nas diretrizes metodológicas do Ministério da Saúde para elaboração de parecer técnico científico com as seguintes informações: características do estudo (desenho, método de coleta e análise); participantes; exposição e custos (Brasil, 2014). A seleção foi comparada para precisão e quaisquer discrepâncias foram resolvidas por consenso ou discussão com outro investigador.

\section{Avaliação de risco de viés dos estudos incluídos na análise}

Dois investigadores de forma independente avaliaram o risco de viés de cada estudo inserido. Foi utilizado a ferramenta Newcastle-Ottawa (Lo, Mertz \& Loeb, 2014) para a avaliação de risco de viés em estudos observacionais e plotado no Review Manager (RevMan)(Review Manager, 2014).

\section{Aspectos éticos}

Por tratar-se de estudo secundário da literatura, não foi necessário a aprovação do Comitê de Ética em Pesquisa.

\section{Resultados e Discussão}

\section{Seleção de estudos}

Após pesquisa nas bases de dados eletrônicas de saúde e remoção das duplicatas, foram identificadas 1.084 referências. Treze artigos eram potencialmente elegíveis para inclusão nesta revisão e, portanto, foram lidos na íntegra. Após leitura e análise crítica, foram selecionados oito artigos para análise qualitativa e quantitativa (metanálise). A literatura cinzenta não reportou achados de acordo com os critérios de elegibilidade. (Figura 1). 
Figura 1 - Diagrama de fluxo e seleção dos estudos incluídos:
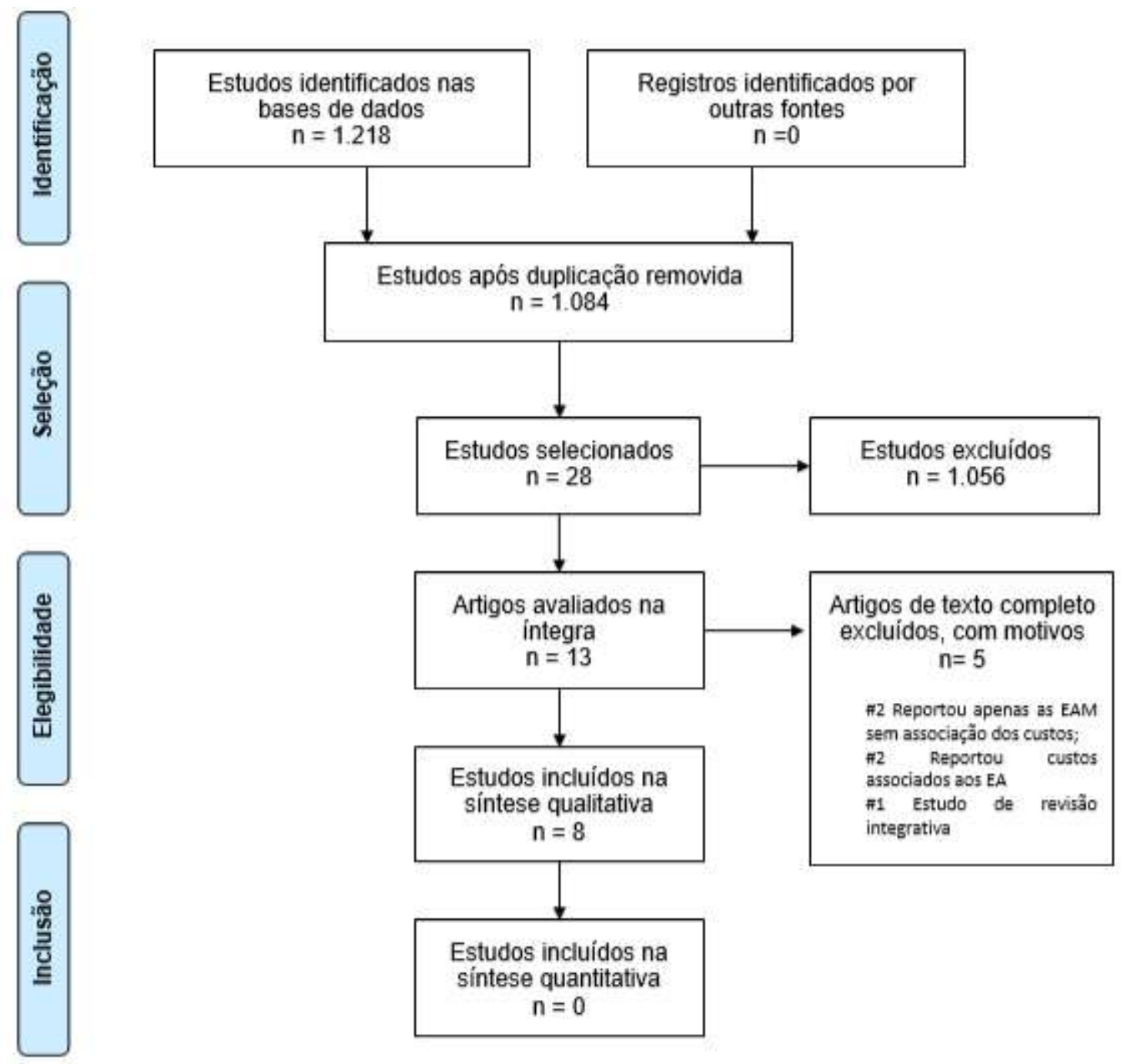

Elaboração: Autores (2020),

Após a leitura na íntegra, oito estudos preencheram os critérios de inclusão e foram inseridos na presente revisão sistemática (Evans et al., 1993; Cullen, et al., 1997; Lagnaoui et al., 2000; Pirmohamed et al., 2004; Hug et al., 2012; Du et al., 2013; Nazer et al., 2013; Parihar et al., 2017). Cinco estudos foram excluídos, sendo dois excluídos por reportarem apenas as EAM sem os custos associados (Rozenfeld, Giordani \& Coelho, 2013; Gutiérrez-Mendoza et al., 2015), dois estudos reportaram os custos associados aos EA de modo geral (Porto et al., 2010; Camargo et al., 2011) e um artigo era revisão integrativa (Vilela et al., 2018).

\section{Descrição dos estudos}

Os oitos estudos observacionais incluídos na análise foram publicados de 1993 a 2017 sendo a maioria americano (cinco estudos) (Evans et al., 1993; Cullen, et al., 1997, Hug et al., 2012; Du et al., 2013, Parihar et al., 2017) um Francês (Lagnaoui et al., 2000), um do Reino Unido (Pirmohamed et al., 2004) e um do oriente médio (Nazer et al., 2013).

A Tabela 1 descreve a caracterização dos estudos incluídos na análise: 
Research, Society and Development, v. 10, n. 4, e21510414030, 2021

(CC BY 4.0) | ISSN 2525-3409 | DOI: http://dx.doi.org/10.33448/rsd-v10i4.14030

Tabela 1 - Caracterização dos estudos incluídos na análise:

\begin{tabular}{|c|c|c|c|}
\hline Autores & $\begin{array}{l}\text { País de } \\
\text { origem }\end{array}$ & Título do Artigo & $\begin{array}{l}\text { Revista/Ano da } \\
\text { Publicação }\end{array}$ \\
\hline $\begin{array}{c}\text { Evans RS, Classen DC, Stevens LE, Pestotnik SL, } \\
\text { Gardner RM, Lloyd JF, Burke JP }\end{array}$ & EUA & $\begin{array}{l}\text { Using a hospital information system to assess the effects } \\
\text { of adverse drug events }\end{array}$ & $\begin{array}{c}\text { Proc Annu Symp } \\
\text { Comput Appl Med Care } \\
1993 \\
\end{array}$ \\
\hline $\begin{array}{c}\text { Cullen, David J, Sweitzer, Bobbie Jean; Bates, } \\
\text { David W, Burdick, Elisabeth; Edmondson } \\
\text { Amy, Leape, Lucian L. }\end{array}$ & EUA & $\begin{array}{c}\text { Preventable adverse drug events in hospitalized patients: } \\
\text { a comparative study of intensive care and general care } \\
\text { units. }\end{array}$ & $\begin{array}{l}\text { Critical Care Medicine } \\
1997\end{array}$ \\
\hline $\begin{array}{c}\text { Lagnaoui R., Moore N, Fach J, Longy-Boursier M, B } \\
\text { Bégaud }\end{array}$ & França & $\begin{array}{c}\text { Adverse drug reactions in a department of systemic } \\
\text { diseases-oriented internal medicine: prevalence, } \\
\text { incidence, direct costs and avoidability }\end{array}$ & $\begin{array}{l}\text { European Journal of } \\
\text { Clinical pharmacology } \\
2000 \\
\end{array}$ \\
\hline $\begin{array}{c}\text { Pirmohamed M, James S, Shaun M, Green C, Scott } \\
\text { AK, Walley TJ, Farrar K, Park BK, Breckenridge } \\
\text { AM. }\end{array}$ & $\begin{array}{l}\text { Reino } \\
\text { Unido }\end{array}$ & $\begin{array}{l}\text { Adverse drug reactions as cause of admission to } \\
\text { hospital: prospective analysis of } 18820 \text { patients. }\end{array}$ & $\begin{array}{c}\text { The BMJ } \\
2004\end{array}$ \\
\hline $\begin{array}{c}\text { Hug BL, Keohane C, Seger DL, Yoon C \& Bates } \\
\text { DW. }\end{array}$ & EUA & $\begin{array}{l}\text { The costs of adverse drug events in community } \\
\text { hospitals. }\end{array}$ & $\begin{array}{c}\text { Jt Comm J Qual Patient } \\
\text { Saf } \\
2012 \\
\end{array}$ \\
\hline $\begin{array}{c}\text { Victoria Tutag Lehr Pharmd, Mary Caverly, Lauren } \\
\text { Kelm, Jaxk Reeves, Mary Lieh- Lai, }\end{array}$ & EUA & $\begin{array}{l}\text { Incidence and costs of adverse drug reactions in a } \\
\text { tertiary care pediatric intensive care unit }\end{array}$ & $\begin{array}{l}\text { American College of } \\
\text { Clinical Pharmacology } \\
2013 \\
\end{array}$ \\
\hline Nazer LH, Eljaber R, Rimawi D \& Hawari FI & $\begin{array}{l}\text { Oriente } \\
\text { médio }\end{array}$ & $\begin{array}{l}\text { Adverse drug events resulting in admission to the } \\
\text { intensive care unit in oncology patients: Incidence, } \\
\text { characteristics and associated cost. }\end{array}$ & $\begin{array}{c}\text { Journal of Oncology } \\
\text { Pharmacy Practice } \\
2013 \\
\end{array}$ \\
\hline $\begin{array}{c}\text { Harish S. Parihar, Hongjun Yin, Jennifer L. Gooch, } \\
\text { Shari Allen, Samuel John,Jianwei Xuan }\end{array}$ & EUA & $\begin{array}{l}\text { Trends in hospital admissions due to antidepressant- } \\
\text { related adverse drug events from } 2001 \text { to } 2011 \text { in the } \\
\text { U.S }\end{array}$ & $\begin{array}{c}\text { BMC Health Services } \\
\text { Reseach } \\
2017 \\
\end{array}$ \\
\hline
\end{tabular}

Fonte: Autores (2020). 
Tabela 2 - Descrição dos objetivos, tipo de estudo/população, custos e síntese dos resultados dos estudos incluídos na análise.

\begin{tabular}{|c|c|c|c|c|}
\hline & Objetivo & Tipo de estudo/população & Custos & Síntese dos Resultados \\
\hline $\begin{array}{l}\text { Evans et al., } \\
1993\end{array}$ & $\begin{array}{l}\text { Avaliar os EAM em pacientes } \\
\text { hospitalizados e seus custos }\end{array}$ & $\begin{array}{l}\text { Estudo observacional } \\
\text { População: Indivíduos } \\
\text { hospitalizados com ocorrência } \\
\text { de EAM. }\end{array}$ & $\begin{array}{c}\text { pacientes com EAM tiveram um custo médio } \\
\text { de hospitalização de US\$10.584 em } \\
\text { comparação a US \$ 5.350 para aqueles sem } \\
\text { EAMs. } \\
\text { O trabalho estimou que os } 569 \text { casos de EAM } \\
\text { no ano de } 1992 \text { resultou em } 1.104 \text { dias } \\
\text { adicionais de pacientes e um custo de US\$ } \\
1.103 .291 .\end{array}$ & $\begin{array}{l}\text { O tempo médio de hospitalização para } \\
\text { pacientes com EAM foi de } 19 \text { dias em } \\
\text { comparação com } 4,36 \\
\text { dias para pacientes controle pareados, o } \\
\text { atributo } \\
\text { A diferença devida foi de } 1,94 \text { dias entre as } \\
\text { populaçôes. O estudo identificou que além } \\
\text { do aumentos de custos, há aumento do } \\
\text { tempo de hospitalização dos pacientes } \\
\text { acometidos por EAM }\end{array}$ \\
\hline $\begin{array}{l}\text { Cullen, et al., } \\
1997\end{array}$ & $\begin{array}{l}\text { Comparar a frequência e a } \\
\text { prevenibilidade de eventos } \\
\text { adversos a medicamentos e } \\
\text { possíveis eventos adversos a } \\
\text { medicamentos em unidades } \\
\text { de terapia intensiva (UTIs) e } \\
\text { não UTIs. }\end{array}$ & $\begin{array}{l}\text { Estudo de coorte prospectivo } \\
\text { População: Adultos em UTIs e } \\
\text { não UTIs }\end{array}$ & $\begin{array}{l}\text { O custo total adicional de um evento adverso } \\
\text { evitável de medicamentos foi de US } \$ 5.857 \text {, e } \\
\text { os custos médicos e cirúrgicos na UTI foram } \\
\text { comparáveis. }\end{array}$ & $\begin{array}{c}\text { Embora os pacientes de UTI tenham uma } \\
\text { taxa significativamente maior de eventos } \\
\text { adversos evitáveis e potenciais do que os } \\
\text { pacientes de fora da UTI, após o ajuste do } \\
\text { número de medicamentos administrados, } \\
\text { essa taxa não difere mais. Assim, uma } \\
\text { maneira de reduzir eventos adversos } \\
\text { evitáveis de medicamentos é reduzir o } \\
\text { número geral de medicamentos usados nas } \\
\text { UTIs. }\end{array}$ \\
\hline $\begin{array}{l}\text { Lagnaoui et } \\
\text { al., } 2000\end{array}$ & $\begin{array}{l}\text { Avaliar a frequência, função } \\
\text { de risco, capacidade de } \\
\text { evitação e custo de EAM } \\
\text { como causa de admissão } \\
\text { hospitalar }\end{array}$ & $\begin{array}{l}\text { Estudo de coorte baseado } \\
\text { realizado em um unidade de } \\
\text { medicina interna por um } \\
\text { período de quatro meses. }\end{array}$ & $\begin{array}{l}\text { O custo dos EAM que levam à hospitalização } \\
\text { foi estimado em } 11.357 \text { euros por leito } \\
\text { hospitalar por ano }\end{array}$ & $\begin{array}{l}\text { EAM em pacientes hospitalizados são } \\
\text { comuns e frequentemente evitáveis. Deve } \\
\text { haver estratégias de prevenção devem ter } \\
\text { como alvo preferencial os prestadores de } \\
\text { cuidados primários de saúde. }\end{array}$ \\
\hline $\begin{array}{l}\text { Pirmohamed } \\
\text { et al., } 2004\end{array}$ & $\begin{array}{l}\text { Verificar a carga atual de } \\
\text { reações adversas a } \\
\text { medicamentos (RAMs) por } \\
\text { meio de uma análise } \\
\text { prospectiva de todas as } \\
\text { internações no hospital. }\end{array}$ & $\begin{array}{c}\text { Estudo observacional } \\
\text { prospectivo } \\
\text { População: pacientes com } \\
\text { idade> } 16\end{array}$ & $\begin{array}{l}\text { O custo anual projetado dessas admissões para } \\
\text { o EAM é de } £ 466 \text { milhões ( } € 706 \text { milhões, US } \\
\$ 847 \text { milhões). }\end{array}$ & $\begin{array}{c}\text { Foram realizadas } 1225 \text { admissões } \\
\text { relacionadas a uma RAM, com uma } \\
\text { prevalência de } 6,5 \% \text {, com a RAM levando } \\
\text { diretamente à admissão em } 80 \% \text { dos } \\
\text { casos. A permanência mediana no leito foi } \\
\text { de oito dias, representando } 4 \% \text { da } \\
\text { capacidade do leito hospitalar. A } \\
\text { fatalidade geral foi de } 0,15 \% \text {. A maioria } \\
\text { das reações foi definitiva ou possivelmente } \\
\text { evitável. }\end{array}$ \\
\hline $\begin{array}{l}\text { Hug et al., } \\
2012\end{array}$ & $\begin{array}{l}\text { Avaliar a custos adicionais e } \\
\text { tempo de permanência em } \\
\text { pacientes com EAM. }\end{array}$ & $\begin{array}{c}\text { Estudo de coorte retrospectivo } \\
\text { multicêntrico realizado em seis } \\
\text { hospitais comunitários com } \\
100 \text { a } 300 \text { leitos durante um } \\
\text { período de observação de } 20 \\
\text { meses }\end{array}$ & $\begin{array}{c}\text { Custo médio de hospitalização não ajustado } \\
\text { aumentou de US\$ } 12.038 \text { em pacientes EAM } \\
\text { com risco de vida (em comparação com apenas } \\
\text { US\$ } 6.804 \text { na população geral de pacientes), o } \\
\text { custo médio ajustado de hospitalização foi um } \\
\text { pouco menor variando US \$ } 9.768 \mathrm{em} \\
\text { pacientes com EAM significativos a } 15.033 \\
\text { dólares em pacientes com EAM com risco de } \\
\text { vida- em comparação com US \$ } 6.917 \text { na } \\
\text { coorte geral. }\end{array}$ & $\begin{array}{c}\text { EAM em hospitais de médio porte } \\
\text { causaram custos consideráveis e perdas } \\
\text { adicionais. } \\
\text { Esses dados fornecem informações úteis } \\
\text { para os hospitais, considerando } \\
\text { investimentos nessa área para melhorar a } \\
\text { segurança de seus cuidados }\end{array}$ \\
\hline $\begin{array}{l}\text { Du et al., } \\
2013\end{array}$ & $\begin{array}{l}\text { Avaliar a incidência, fatores } \\
\text { de risco e carga econômica de } \\
\text { EAM na Unidade de Terapia } \\
\text { Intensiva Pediátrica. }\end{array}$ & $\begin{array}{l}\text { Estudo prospectivo População: } \\
\text { todos os pacientes entre } 1 \text { e } 18 \\
\text { anos de idade internados na } \\
\text { unidade de Unidade de } \\
\text { Terapia Intensiva Pediátrica } \\
\text { (UTIP). }\end{array}$ & $\begin{array}{c}\text { Os custos médios e medianos das instalações } \\
\text { da UTIP foram de US\$18.993 e US \$ } 7.720 \text {, } \\
\text { com intervalo interquartil de US \$ } 3.561 \text { a US } \\
\$ 18.738 \text {. O custo total médio de uma admissão } \\
\text { na UTIP sem EAM's foi de US \$ } 11.860 \pm \text { US } \\
\$ 14.040 \text { (DP) em comparação com US \$ } \\
62.616,7 \pm \text { US \$ 9.309 para uma admissão com } \\
\text { EAM. }\end{array}$ & $\begin{array}{l}\text { Deve haver programas de monitoramento } \\
\text { específicos na UTIP. Idade menor, alta } \\
\text { gravidade da doença, cirurgia } \\
\text { cardiovascular e polifarmácia foram } \\
\text { associados a ocorrência de EAM na UTIP. } \\
\text { O aumento da vigilância é uma possível } \\
\text { intervenção para melhorar os resultados em } \\
\text { pacientes de UTIP e reduzir os custos da } \\
\text { unidade. }\end{array}$ \\
\hline $\begin{array}{l}\text { Nazer et al., } \\
2013\end{array}$ & $\begin{array}{l}\text { Descrever a incidência, } \\
\text { características e custo de } \\
\text { eventos adversos a } \\
\text { medicamentos que requerem } \\
\text { admissão na unidade de } \\
\text { terapia intensiva em pacientes } \\
\quad \text { oncológicos }\end{array}$ & $\begin{array}{l}\text { Estudo observacional } \\
\text { prospectivo População: } \\
\text { Pacientes oncológicos }\end{array}$ & $\begin{array}{l}\text { O valor total dos pacientes que apresentram } \\
\text { EAM foi de US\$ 631.380. O custo médio para } \\
\text { o gerenciamento de uma admissão relacionada } \\
\text { à EAM na UTI foi de US } \$ 11.692 \pm 17.529 \\
\text { (DP) e o custo médio foi de US } \$ 5.385 \\
\text { (variação de } 383 \text { a } 99.234 \text { ). Com base nos } \\
\text { dados deste estudo, estimamos que haveria } \\
\text { cerca de } 11 \text { internações por mês relacionadas a } \\
\text { EAM, o que corresponde a aproximadamente } \\
\text { US } \$ 1,5 \text { milhão em taxas anuais } \\
\end{array}$ & $\begin{array}{c}\text { O estudo constatou que eventos adversos a } \\
\text { medicamentos foram causa significativa de } \\
\text { internações na UTI e mortalidade. Além } \\
\text { disso, as internações na UTI relacionadas a } \\
\text { medicamentos foram associadas à } \\
\text { utilização significativa de recursos e } \\
\text { aumento dos custos. }\end{array}$ \\
\hline $\begin{array}{l}\text { Parihar et al., } \\
2017\end{array}$ & $\begin{array}{l}\text { Examinar as mudanças nas } \\
\text { internações devido a EAM } \\
\text { relacionadas a antidepressivos } \\
\text { entre diferentes grupos } \\
\text { sociodemográficos e } \\
\text { alterações no tempo de } \\
\text { permanência } \\
\end{array}$ & $\begin{array}{l}\text { Análise retrospectiva dos } \\
\text { dados secundários com } \\
\text { inclusão de população a partir } \\
\text { de } 18 \text { anos de idade. }\end{array}$ & $\begin{array}{c}\text { O tempo médio de permanência para todas as } \\
\text { internações relacionadas ao EAM aumentou de } \\
2,18 \text { para } 2,81 \text { dias e as despesas médicas do } \\
\text { hospital aumentaram de US\$ } 8.456,2 \text { para US\$ } \\
21.572,5 \text {. }\end{array}$ & $\begin{array}{c}\text { As admissões hospitalares relacionadas a } \\
\text { antidepressivos especialmente em idosos } \\
\text { incorreram em uma carga econômica maior } \\
\text { para a sociedade }\end{array}$ \\
\hline
\end{tabular}

Fonte: Autores (2020).

\section{Avaliação do risco de viés}

A avaliação do risco de viés dos estudos incluídos no presente estudo demonstrou que no domínio igualdade entre as coortes expostas e não expostas serem retiradas da mesma população há quatro estudos com baixo risco (Evans et al., 1993, Lagnaoui et al., 2000, Du et al., 2013, Nazer et al., 2013) e quatro estudos com alto risco de viés, pois um estudo 
comparou EAM em pacientes de UTI versus não UTI (Cullen, et al., 1997), um estudo comparou pacientes cm EAM em todas as unidades de sua dependência (Pirmohamed et al., 2004), outro estudo era multicêntrico (Hug et al., 2012) e o outro comparou EAM a antidepressivos em todas as idades e populações (Parihar et al., 2017).

No domínio confiança na avaliação da exposição, há cinco estudos com baixo risco (Evans et al., 1993; Cullen, et al., 1997; Lagnaoui et al., 2000, Du et al., 2013, Nazer et al., 2013) e três estudos incertos pois um abrangeu todas as internações no hospital (Pirmohamed et al., 2004), um foi multicêntrico (Hug et al., 2012) e um oriundo de dados secundários (Parihar et al., 2017).

No domínio confiança de que o resultado dos desfechos não estavam presente no início os oito estudos foram classificados como baixo risco (Evans et al., 1993; Cullen, et al., 1997; Lagnaoui et al., 2000; Pirmohamed et al., 2004; Hug et al., 2012; Du et al., 2013; Nazer et al., 2013; Parihar et al., 2017).

No quesito demonstração do grupo exposto e não exposto para todas as variáveis estão associadas ao desfecho de interesse ou a análise estatística ajustou para essas variáveis prognósticas demonstrou que sete estudos (Evans et al., 1993; Cullen, et al., 1997; Lagnaoui et al., 2000; Pirmohamed et al., 2004; Hug et al., 2012; Du et al., 2013; Nazer et al., 2013) apresentaram baixo risco de viés e um estudo incerto, pois utilizou dados secundários (Parihar et al., 2017).

No domínio confiança na avaliação da presença ou ausência de fatores prognósticos os oito estudos foram classificados como baixo risco (Evans et al., 1993; Cullen, et al., 1997; Lagnaoui et al., 2000; Pirmohamed et al., 2004; Hug et al., 2012; Du et al., 2013; Nazer et al., 2013; Parihar et al., 2017).

No domínio confiança na avaliação do resultado os oito estudos foram classificados como baixo risco (Evans et al., 1993; Cullen, et al., 1997; Lagnaoui et al., 2000; Pirmohamed et al., 2004; Hug et al., 2012; Du et al., 2013; Nazer et al., 2013; Parihar et al., 2017).

No acompanhamento adequado das coortes, os oito estudos foram classificados como baixo risco (Evans et al., 1993; Cullen, et al., 1997; Lagnaoui et al., 2000; Pirmohamed et al., 2004; Hug et al., 2012; Du et al., 2013; Nazer et al., 2013; Parihar et al., 2017).

Finalmente no domínio das intervenções semelhantes entre os grupos, cinco foram classificados como baixo risco (Evans et al., 1993; Cullen, et al., 1997; Lagnaoui et al., 2000, Du et al., 2013, Nazer et al., 2013) e três estudos como incertos, pois um pois um abrangeu todas as internações no hospital (Pirmohamed et al., 2004), um foi multicêntrico (Hug et al., 2012), um estudo comparou EAM a antidepressivos em todas as idades e populações (Parihar et al., 2017). As figuras de risco de viés encontram-se sumarizados na Figura 2. 
Research, Society and Development, v. 10, n. 4, e21510414030, 2021

(CC BY 4.0) | ISSN 2525-3409 | DOI: http://dx.doi.org/10.33448/rsd-v10i4.14030

Figura 2. Risco de viés dos estudos inseridos na análise.

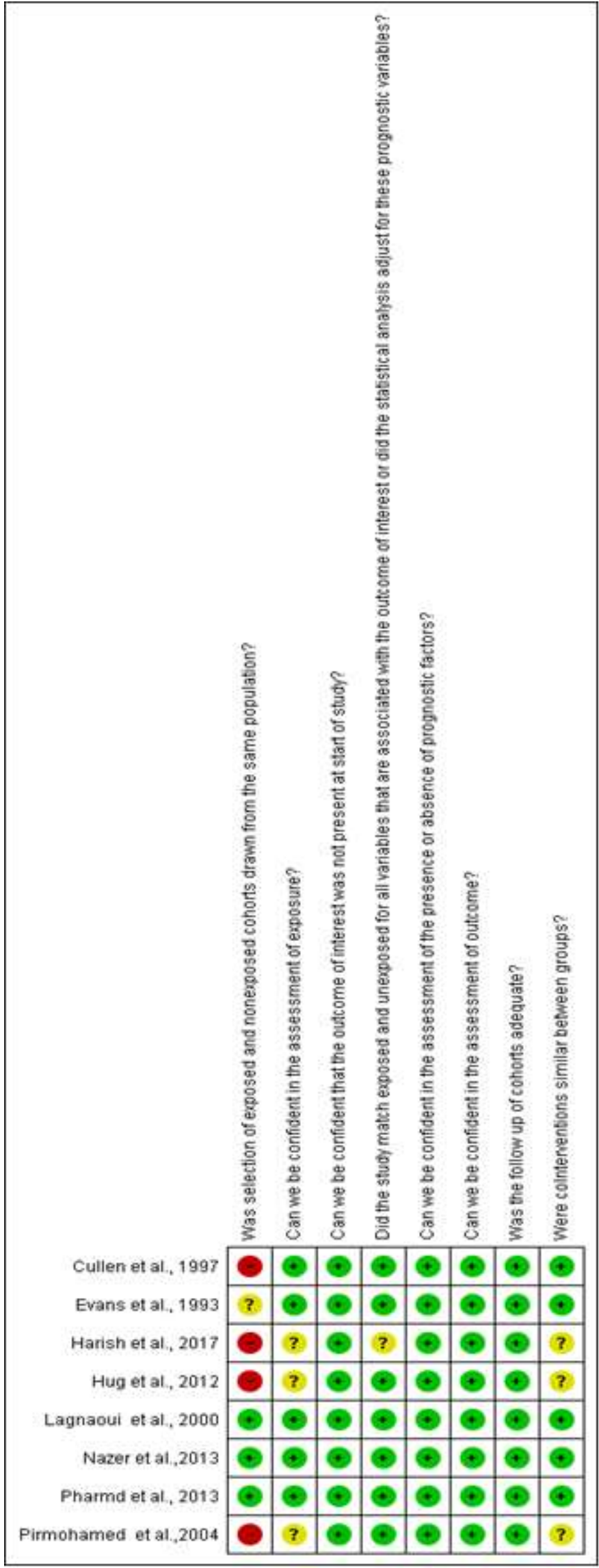

Interpretação dos Resultados e Conclusão. Fonte: Autores (2020). 


\section{Sumário da evidência}

Na literatura, esta é a primeira revisão sistemática com o objetivo de identificar os custos hospitalares associados a ocorrência de evento adverso medicamentoso (EAM).

Um estudo realizado em hospital de grande porte americano revelou que os indivíduos hospitalizados com ocorrência de EAM tiveram um custo médio de hospitalização de US\$ 10.584 em comparação a US\$ 5.350 para aqueles sem EAMs (Evans et al., 1993)

Um outro trabalho realizado em hospital de médio porte americano estimou um custo adicional de US\$1.103.291 anual na ocorrência de EAMs (Lagnaoui et al., 2000). Um outro estudo revelou que o custo médio de hospitalização não ajustado aumentou de US\$ 12.038 em pacientes EAM com risco de vida (em comparação com apenas US\$ 6.804 na população geral de pacientes) e o custo médio ajustado de hospitalização foi um pouco menor variando US\$ $9.768 \mathrm{em}$ pacientes com EAM significativos a 15.033 dólares em pacientes com EAM com risco de vida- em comparação com US\$ 6.917 na coorte geral (Hug et al., 2012).

De modo geral, o tempo médio de hospitalização para pacientes com EAM foi de 19 dias em comparação com 4,36 dias para pacientes controle pareados. Deste modo, fica evidente que além dos aumentos de custos, há aumento do tempo de hospitalização dos pacientes acometidos por EAM e que deve haver estratégias de prevenção devem ter como alvo preferencial os prestadores de cuidados primários de saúde (Evans et al., 1993; Cullen, et al., 1997; Lagnaoui et al., 2000).

Nas UTI's a análise demonstrou que o custo médio para o gerenciamento de uma admissão relacionada à EAM na UTI foi de US\$ $11.692 \pm 17.529$ (DP) e o custo médio foi de US\$ 5.385 (variação de 383 a 99.234). Com base nos dados deste estudo, estimamos que haveria cerca de 11 internações por mês relacionadas a EAM, o que corresponde a aproximadamente US\$ 1,5 milhão em taxas anuais de pacientes para uma instituição de câncer com 170 leitos e uma UTI para adultos com 12 leitos (Nazer et al., 2013). Já outro estudo revelou que o custo total adicional de um evento adverso evitável de medicamentos foi de US\$ 5.857, e os custos médicos e cirúrgicos na UTI foram comparáveis (Cullen, et al., 1997).

Na UTI pediátrica o custo total médio de uma admissão na UTIP sem EAM’s foi de US \$ $11.860 \pm$ US \$14.040 (DP) em comparação com US \$ 62.616,7 \pm US\$ 9.309 para uma admissão com EAM (Du et al., 2013).

Um estudo que teve na análise o foco nas admissões hospitalares relacionadas a antidepressivos identificou que o tempo médio de permanência para todas as internações relacionadas ao EAM aumentou de 2,18 para 2,81 dias e as despesas médicas do hospital aumentaram de US\$ 8.456,2 para US\$21.572,5 (Parihar et al., 2017).

\section{Limitações}

A presente revisão sistemática possui limitações, sendo a principal relacionada a variabilidade dos locais que os estudos foram realizados, deste modo, impedindo que os dados fossem agrupados para uma análise quantitativa. Porém não foi selecionado um único local por desejar obter visão geral do que há na literatura associado a ocorrência de EAM’s.

\section{Conclusão}

\section{Implicações para prática e para pesquisas futuras}

Foi possível obter um panorama referente aos custos gerados em decorrência dos EAM nas instituições de saúde por meio da sumarização dos artigos disponíveis na literatura. A literatura evidenciou que há intenso aumento dos custos associados a ocorrência de EAM, bem como consequências negativas como aumento do tempo de internação, necessidade de 
mão de obra especializada e exposição a danos do paciente.

A análise de viés dos estudos demonstrou que eles apresentam limitações metodológicas, evidenciando a necessidade de mais estudos na área com a adoção de métodos rigorosos na elaboração. Esta análise poderá ser um poderoso instrumento gerencial para as instituições de saúde como demonstrativo dos custos decorrentes dos eventos adversos medicamentosos, bem como fornece subsidio para pesquisas futuras.

\section{Agradecimentos}

A Universidade de Guarulhos e ao Programa Institucional de Bolsa de Iniciação Científica (PIBIC) CNPq pelo financiamento da pesquisa.

\section{Referências}

Abreu, C. C. F., Rodrigues, M. A. \& Paixão, M. P. B. A. (2013). Erros de medicação reportados pelos enfermeiros da prática clínica. Rev. Enf. Ref., Coimbra, $\operatorname{serIII}(10), 63-8$.

Anvisa. Notivisa - Sistema Nacional de Notificações para a Vigilância Sanitária. Manual do Usuário. (2013). Anexo 3 Formulário para Notificação de Eventos Adversos à Medicamento - Profissional da Saúde.

Araújo, A. S. S. C. \& Mello, J. M. H. P. (2017). Internações hospitalares por lesões decorrentes de acidente de transporte terrestre no Brasil, 2013: permanência e gastos. Epidemiol. Serv. Saúde. 26(1): 31-8.

Brasil. Ministério da Saúde. (2013). Introdução à Gestão de Custos em Saúde / Ministério da Saúde, Organização Pan-Americana da Saúde. - Brasília : Editora do Ministério da Saúde, 148 p

Brasil. (2014). Ministério da Saúde. Secretaria de Ciência, Tecnologia e Insumos Estratégicos. Departamento de Ciência e Tecnologia. Diretrizes metodológicas: elaboração de pareceres técnico-científicos / Ministério da Saúde, Secretaria de Ciência, Tecnologia e Insumos Estratégicos, Departamento de Ciência e Tecnologia. (4a ed.), Ministério da Saúde, 80 p.

Camargo, A. E. B. S., et al. (2011). Eventos adversos a medicamentos em um hospital sentinela do Estado de Goiás, Brasil. Revista latino-americana de enfermagem, 19(2).

Carvalho, N. G. G. de, Assunção, M. V. D. de, Fernandes, D. R. M., \& Silveira, M. L. da S. S. (2020). Gestão de custos como instrumento de eficiência no âmbito do Ministério Público do Estado do Rio Grande do Norte. Research, Society and Development, 9(8), e412985473.

Cullen, D. J., Sweitzer, B. J., Bates, D. W., Burdick, E. \& Edmondson, A., Leape, L. L. (1997). Preventable adverse drug events in hospitalized patients: a comparative study of intensive care and general care units. Crit Care Med. 25(8):1289-97.

Du, W., Tutag, L. V., Caverly, M., Kelm, L., Reeves, J. \& Lieh-Lai, M. (2013). Incidence and costs of adverse drug reactions in a tertiary care pediatric intensive care unit. J Clin Pharmacol. 53(5):567-573.

Evans, R. S., Classen, D. C., Stevens, L. E., Pestotnik, S. L., Gardner, R. M., Lloyd, J. F. \& Burke, J. P. (1993). Using a hospital information system to assess the effects of adverse drug events. Proc Annu Symp Comput Appl Med Care.161-5.

Fernandes, A. M. M. L. \& Queiros, P. J. P. (2011). Cultura de Segurança do Doente percecionada por enfermeiros em hospitais distritais portugueses. Rev. Enf. Ref., 4, 37-48.

Fonteles, M. M. F. et al. (2009). Reações adversas causadas por fármacos que atuam no sistema nervoso: análise de registros de um centro de farmacovigilância do Brasil. Rev Psiq Clín.36(4):137-44.

Giordani, F. et al. (2012). Vigilância de eventos adversos a medicamentos em hospitais: aplicação e desempenho de rastreadores. Rev Bras Epidemiol. 15(3): 455-67.

Gutiérrez-Mendoza, L. M. et al. (2015). Costos de eventos adversos graves en un Hospital Comunitario de Ensenanza en México. Cirugía y Cirujanos.83(3):211-216.

Hug, B. L., Keohane, C. \& Seger, D. L., et al. (2012) The costs of adverse drug events in community hospitals. Jt Comm J Qual Patient Saf. 38(3):120-6.

Lagnaoui, R., Moore, N., Fach, J., Longy-Boursier, M. \& Begaud, B. (2000).Adverse drug reactions in a department of systemic diseases-oriented internal medicine: prevalence, incidence, direct costs and avoidability.Eur J Clin Pharmacol. 56:181-6.

Lo, C. K-L., Mertz, D., \& Loeb, M.(2014). Newcastle-Ottawa Scale: comparing reviewers' to authors' assessments. BMC Medical Research Methodology. 14:45. 24690082

Moher, D., Shamseer, L., Clarke, M., Ghersi, D., Liberati, A., \& Petticrew, M. PRISMA-P Group (2015). Preferred reporting items for systematic review and meta-analysis protocols (PRISMA-P) 2015 statement. Systematic Review, 4(1),1. 10.1186/2046-4053-4-1 
Research, Society and Development, v. 10, n. 4, e21510414030, 2021

(CC BY 4.0) | ISSN 2525-3409 | DOI: http://dx.doi.org/10.33448/rsd-v10i4.14030

Moreira, A, Bezerra, A. L. Q, Paranaguá, T. T. B., Silva, A. E. B. C \& Filho. F. M. A. (2015). Conhecimento dos profissionais de saúde sobre eventos adversos em unidade de terapia intensiva. Rev enferm UERJ, 23(4):461-7.

Nazer, L. H., Eljaber, R., Rimawi, D. \& Hawari, F. (2013). Adverse drug events resulting in admission to the intensive care unit in oncology patients: Incidence, characteristics and associated cost. J Oncol Pharm Pract. 19(4):298-304.

Parihar, H. S., Yin, H., Gooch, J. L. et al. (2017). Trends in hospital admissions due to antidepressant-related adverse drug events from 2001 to 2011 in the U.S. BMC Health Serv Res, 17: 51.

Pena M. M \& Melleiro M. M.(2018). Eventos adversos decorrentes de falhas de comunicação: reflexões sobre um modelo para transição do cuidado. Rev Enferm UFSM. 8(3):616- 25

Pirmohamed, M., James, S. M. S. et al. (2004). Adverse drug reactions as cause of admission to hospital: prospective analysis of 18820 patients. BMJ. 329 (7456) 15- 19.

Porto, S. et al. (2010). A magnitude financeira dos eventos adversos em hospitais no Brasil. Rev Port Saúde Pública.10: 74-80.

Porto, S., Martins, M., Mendesa, W. \& Travassos, C. (2010). A magnitude financeira dos eventos adversos em hospitais no Brasil. Rev Port Saúde Pública.(10):74-80

Review Manager (RevMan) (2014).[Computer program]. Version 5.3. Copenhagen: The Nordic Cochrane Centre, The Cochrane Collaboration.

Roque, K. E, \& Melo, E. C. P. (2012). Avaliação dos eventos adversos a medicamentos no contexto hospitalar. Escola Anna Nery Revista de Enfermagem. 16(1), 121-127.

Rozenfeld, S. A (2007). gravos provocados por medicamentos em hospitais do Estado do Rio de Janeiro, Brasil. Rev Saude Publica. 41(1):108-115.

Rozenfeld, S., Giordani, F., \& Coelho, S. (2013). Eventos adversos a medicamentos em hospital terciário: estudo piloto com rastreadores. Revista de Saúde Pública, 47: 1102-1111.

Silva, E. N., Silva, M. T. \& Pereira, M. G. (2016). Estudos de avaliação econômica em saúde: definição e aplicabilidade aos sistemas e serviços de saúde. Epidemiol Serv Saude. 25(1):205-7

Silva, E. N., Silva, M. T \& Pereira, M. G. (2016). Identificação, mensuração e valoração de custos em saúde. Epidemiol Serv Saude.25 (2):437-39

Stroup, D. F., Berlin, J. A ., Morton, S. C ., Olkin, I ., Williamson, G. D ., Rennie, D, et al. (2000). Meta-analysis of observational studies in epidemiology: a proposal for reporting. Meta-analysis Of Observational Studies in Epidemiology (MOOSE) group. JAMA, 283(15), 2008- 12.

Vilela, R. P. B. et al. (2018). Custo do erro de medicação e eventos adversos à medicação na cadeia medicamentosa: uma revisão integrativa. JBES: Brazilian Journal of Health Economics/Jornal Brasileiro de Economia da Saúde, 10.2. 